\title{
INFLUENCIA DE LA CAL EN EL MORTERO PARA PEGA DE BLOQUES
}

\author{
Alejandro Navas Carro \\ Catherine M. Acón Villalobos
}

\begin{abstract}
Resumen
La finalidad de este estudio fue comprobar, experimentalmente, si el uso de cal en la mezcla para el mortero para pega de bloques de mampostería trae beneficios en sus propiedades. Se obtuvieron los porcentajes de retención de agua y contenido de aire de los morteros y se compararon con las especificaciones de la norma ASTM C270. Asimismo, se determinó la resistencia a la compresión a los 7, 28 y 56 días; la resistencia a los 28 días se comparó con la norma ASTM C270. En primer lugar se visitaron caleras en Costa Rica, se realizaron pruebas químicas a las cales, se determinó su granulometría y retención de agua. Estas pruebas iniciales sirvieron como criterio para seleccionar las cales utilizadas en la mezcla del mortero, con la finalidad de probar su influencia en las propiedades del mortero en estado fresco y endurecido. Por medio de las pruebas realizadas, se pudo comprobar que el uso de cal en la mezcla del mortero para pega de bloques trae beneficios en los parámetros evaluados en este estudio. Se obtuvieron mejoras en los porcentajes de retención de agua, contenido de aire y la resistencia a la compresión, debido a esto se recomienda su uso.
\end{abstract}

Palabras clave: Cal; propiedades del mortero; pega de bloques en mampostería; retención de agua; contenido de aire; resistencia

\begin{abstract}
Abstrat
The purpose of this study was to test out experimentally whether the use of lime in the mixture of mortar for masonry block pasting brings along benefits on its properties. Water retention percentages and air content of the mortar were obtained and compared to the specifications of the ASTM C270. Compressive strength at the age of 7, 28 and 56 days were tested; the results of strength at the age of 28 days were compared with the ASTM C270.

Costa Rican limestone quarries with kilns were visited, chemical tests were applied to the limes, sieve analysis and water retention were determined as well. These initial tests were used as criteria to select the limes used in the mortar mixture in order to test their influence in the properties of the mortar in fresh and hardened state.

Through tests carried out, it was found that the use of lime in mortar mixture for block pasting brings benefits under the parameters evaluated in this study. Improvements were obtained in water retention, air content, and compressive strength; therefore its use is recommended.
\end{abstract}

Keywords: Lime; mortar properties; block pasting in masonry; water retention; air content; resistance.

Recibido: 02 de Octubre 2014 Aprobado: 16 de febrero 2015

\section{INTRODUCCIÓN}

Actualmente, en Costa Rica no se suele utilizar la cal para la mezcla de mortero para pega de bloques en mampostería preparada en sitio, por desconocimiento de los posibles efectos que esta pueda ocasionar. Esta investigación tiene la finalidad de verificar si el uso de la cal brinda las mismas ventajas reportadas en otros países, entre ellos Estados Unidos, Alemania, Italia y México.

Primero, es importante tener en cuenta la utilidad de un mortero de pega para mampostería, 
su función y la de sus componentes. Entre las propiedades que debe presentar un mortero para pega se encuentran: la fácil colocación, el soporte del peso de las unidades de mampostería, adherencia a la superficie en la que se aplica, buena trabajabilidad y retención de agua. Según el estudio de Thomson (2000) y demás estudios realizados en otros países, la cal suele brindar mejoras en cuanto a trabajabilidad y plasticidad. Además, permite que aumente el contacto entre las partículas del agregado fino, evitando así la contracción durante el proceso de secado. Es importante mencionar que, a través de los años, en morteros con cal agregada, se va llevando a cabo un proceso químico conocido como carbonatación, el cual permite que se auto sellen las grietas pequeñas formadas a través del tiempo. Esta reacción química no se logra con los aditivos que hay actualmente para los morteros en Costa Rica.

Con respecto al tema de la influencia de la cal en morteros, se tienen estudios que evalúan como parte de la investigación el efecto de la cal en el mortero para repello, por ejemplo los trabajos de Picado (2001) y Chacón (2001). Sin embargo, no se encontraron estudios específicos para evaluar las propiedades que brinda la cal al ser utilizada en morteros para pega de bloques en mampostería.

El estudio tiene como objetivo general, estudiar la influencia de la cal en las propiedades del mortero para pega de bloques de mampostería. Específicamente, se busca evaluar los efectos de la cal en los porcentajes de retención de agua, contenido de aire y la resistencia a la compresión a los 7, 28 y 56 días del mortero de pega.

La relevancia de este estudio se basa en la posibilidad de encontrar resultados positivos en las propiedades del mortero que indiquen que la producción de cal hidratada en Costa Rica se podría ampliar al mercado de la construcción.

\subsection{Aporte}

El estudio busca determinar si el uso de cal en la preparación del mortero de pega de bloques de mampostería brinda beneficios en las propiedades del mortero, específicamente retención de agua, contenido de aire y resistencia a la compresión. Estas propiedades son de importancia pues permiten aumentar su calidad, trabajabilidad y plasticidad; a su vez, mejorar la adherencia entre el mortero y los bloques de mampostería, de manera que se puedan realizar construcciones en mampostería con mayor facilidad y mejores resultados.

\section{MÉTODOS Y MATERIALES}

La metodología empleada en la realización del trabajo se desarrolló en las siguientes tres etapas.

\section{Etapa bibliográfica e investigación de campo:}

Esta primera parte contempló la revisión de material bibliográfico, con énfasis en las principales propiedades que deben tener los morteros de pega de bloques, ya sean tipo $\mathrm{M}$, $\mathrm{S}$ o $\mathrm{N}$. Asimismo, se llevaron a cabo visitas a diferentes fábricas de cal en el país, donde fue posible observar su proceso de fabricación.

\section{Etapa experimental:}

El proceso experimental y muestreo fue la etapa del proyecto que tomó mayor tiempo debido a que incluyó las pruebas por realizar a las diferentes muestras de cal obtenidas de cada fábrica seleccionada para el estudio.

Para cada muestra obtenida, se realizaron pruebas químicas para determinar los porcentajes de hidróxido de calcio, hidróxido de magnesio y dióxido de carbono. Con estos datos y las granulometrías de las cales se hizo una selección de las muestras más pertinentes por evaluar. En este caso, las muestras fueron seleccionadas tomando en cuenta las cales que presentaron los porcentajes de hidróxido de calcio, hidróxido de magnesio, dióxido de carbono y granulometría más cercanos a lo recomendado por las normas ASTM. Posteriormente, se realizó un segundo muestreo a las industrias seleccionadas para observar el grado de variabilidad que se presentaban en cuanto a composición química y granulometría. Para la fabricación de los especímenes de mortero, se utilizaron las muestras de cal de las industrias seleccionadas correspondientes al primer muestreo.

$\mathrm{Se}$ fabricaron los diferentes tipos de mortero, con cal y sin cal. Para los morteros 
en estado fresco se llevaron a cabo pruebas de retención de agua y contenido de aire, mientras que para los morteros en estado endurecido se realizaron pruebas de resistencia a compresión a las edades de 7, 28 y 56 días.

\section{Análisis de resultados y conclusiones}

La última etapa del trabajo consistió en el análisis de la información adquirida en las pruebas para la redacción de conclusiones y posibles recomendaciones.

A continuación se detallan las propiedades de los materiales utilizados para la fabricación de los especímenes de mortero: el agregado fino, cemento y cal. Asimismo, se indican las proporciones utilizadas para cada tipo de mortero.

\subsection{Materiales}

\section{Agregado fino}

Para la elaboración de los morteros en este estudio, se empleó arena tipo industrial. Dentro de sus características físicas se encontraron: peso unitario suelto de $1006 \mathrm{~kg} / \mathrm{m}^{3}$, peso unitario envarillado de $1124 \mathrm{~kg} / \mathrm{m} 3$, peso específico bruto seco de 2,60, un 2,65\% de absorción y un módulo de finura de 2,36. La granulometría de la arena se presenta en la Tabla 1.

\section{Cemento}

Se usó cemento de producción nacional, de uso general con las siguientes propiedades: peso específico de 3,00 , tiempo de fragua de 140 minutos y resistencia a la compresión de 4,8 $\mathrm{MPa}$.

\section{Cal}

Se investigaron las diferentes fuentes de cal en el país con el fin de definir cuáles se usarían en este estudio. A estas muestras se les realizaron pruebas químicas y se escogieron las que presentaron mayores resultados en los contenidos de hidróxido de calcio, pues la norma ASTM C207-06 indica que la suma de hidróxido de calcio e hidróxido de magnesio deben ser mínimo de 95\%. Las formaciones de donde se escogieron las cales se distribuyen en el territorio nacional: Barra Honda (valle del Tempisque), San Miguel (sur del valle central, Desamparados y Cartago), Las Animas (Turrialba), El Viejo (península de Santa

Tabla 1. Granulometría del agregado fino.

\begin{tabular}{ccc}
\hline Malla & Pasando & Límites pasando (\%) según \\
\hline 4 & 100,0 & ASTM C144 \\
\hline 8 & 92,1 & 100 \\
16 & 67,3 & $95-100$ \\
30 & 49,2 & $70-100$ \\
50 & 34,0 & $40-75$ \\
100 & 21,9 & $20-40$ \\
200 & 13,8 & $10-25$ \\
charola & 0,0 & $0-10$ \\
\hline
\end{tabular}


Elena), Brito (cordillera Costeña), junto con cales provenientes de Guatemala y Honduras. La cal utilizada es lo que se conoce como cal hidratada o cal apagada, es decir, hidróxido de calcio. Las caleras específicas muestreadas en este estudio se muestran en la Tabla 3.

\section{Morteros}

Los morteros se fabricaron según las proporciones indicadas en la Tabla 4. Estas proporciones se basan en el Código Sísmico de Costa Rica 2010 y en el ACI 530-05.

Luego se evaluaron en estado fresco los morteros tipoLuego se evaluaron en estado fresco los morteros tipo $\mathrm{M}, \mathrm{S}$ y $\mathrm{N}$ para su retención de agua y contenido de aire. Finalmente, para evaluarlos en estado endurecido también se tomaron los morteros tipo M, S y N, y se verificó su resistencia a los 7 , 28 y 56 días. A partir de los datos obtenidos en los diferentes ensayos realizados a las muestras, se crearon las Figuras 1 a 4 y las Tablas 6 al 12 que permiten visualizar las mejoras o pérdidas, en las propiedades del mortero, obtenidas al usar cal nacional y cal importada.

\section{RESULTADOS Y DISCUSIÓN}

\subsection{Pruebas a la cal hidratada}

A partir de las pruebas realizadas fue posible demostrar que el uso de la cal nacional puede generar mejoras en el mortero para pega. A continuación se mostrarán los resultados en cada prueba.

En las Tablas 3 y 4 se presentan los resultados obtenidos de las pruebas químicas realizadas a todas las muestras de cal recolectadas en el primer y segundo muestreo, respectivamente. Según la norma ASTM C207-06 (2011), para la cal hidratada normal (hidratación Tipo $\mathrm{N}$, de acuerdo con su denominación en la norma ASTM C207-06(2011)), la suma del porcentaje de hidróxido de calcio más el porcentaje de hidróxido de magnesio debe ser mayor o igual que $95 \%$, así como el porcentaje de dióxido de carbono debe ser menor que $5 \%$. Con el segundo muestreo, se pretendió comparar la variabilidad en las propiedades de las cales nacionales seleccionadas al cambiar la época de obtención de las muestras: el primer muestreo se realizó en verano y el segundo en invierno. Además, las tablas presentan los porcentajes de material retenido en las mallas número 30 y 200 de todas las caleras visitadas para el primer y segundo muestreo. Los resultados se comparan con los indicados en la ASTM C207-06 (2011) en la que se permite no más del $0,5 \%$ retenido en la malla No. 30 .

El primer muestreo indica que las caleras ubicadas en la zona de Guanacaste (Formación Barra Honda) al acercarse en mayor medida a los porcentajes requeridos para el hidróxido de calcio, hidróxido de magnesio y dióxido de carbono, son las que muestran los resultados químicos más adecuados. Se observa que en ningún caso se alcanza el porcentaje mínimo

Tabla 2. Proporciones volumétricas de los morteros usados.

\begin{tabular}{|c|c|c|c|c|}
\hline & \multirow{2}{*}{ Tipo de mortero } & \multicolumn{3}{|c|}{ Proporciones volumétricas del mortero } \\
\hline & & Cemento & Cal hidratada & $\begin{array}{l}\text { Arena en condición } \\
\text { húmeda y suelta }\end{array}$ \\
\hline A & & 1 & $1 / 4$ & 3 \\
\hline B & & 1 & $1 / 2$ & 4 \\
\hline $\mathrm{C}$ & & 1 & $3 / 4$ & 5 \\
\hline
\end{tabular}


Tabla 3. Resultados de pruebas a la cal para el primer muestreo de las caleras visitadas.

\begin{tabular}{|c|c|c|c|c|c|c|c|c|}
\hline \multirow{2}{*}{ Formación } & \multirow{2}{*}{$\#$} & \multirow{2}{*}{ Calera } & \multicolumn{3}{|c|}{ Pruebas químicas } & \multicolumn{2}{|c|}{$\begin{array}{c}\text { Porcentajes } \\
\text { retenidos }\end{array}$} & \multirow{2}{*}{$\begin{array}{c}\begin{array}{c}\text { Reten- } \\
\text { ción de } \\
\text { agua }\end{array} \\
\begin{array}{c}\text { Prome- } \\
\text { dio }\end{array}\end{array}$} \\
\hline & & & $\mathrm{Ca}(\mathrm{OH})_{2}$ & $\mathrm{Mg}(\mathrm{OH})_{2}$ & $\mathrm{CO}_{2}$ & Ret. 30 & Ret. 200 & \\
\hline \multirow{3}{*}{$\begin{array}{l}\text { Barra } \\
\text { Honda }\end{array}$} & 1 & $\begin{array}{l}\text { Industrias } \\
\text { de Calcio }\end{array}$ & 87,3 & 0,9 & 4,9 & $0,0 \%$ & $1,5 \%$ & $86,4 \%$ \\
\hline & 2 & Níspero & 82,8 & 0,9 & 4,3 & $29,8 \%$ & $49,5 \%$ & $89,7 \%$ \\
\hline & 3 & Nicoyana & 91,1 & 0,1 & 2,1 & $0,3 \%$ & $7,9 \%$ & $93,5 \%$ \\
\hline Brito & 4 & $\begin{array}{c}\text { Roca } \\
\text { Piedra } \\
\text { Grande }\end{array}$ & 64,1 & 0,3 & 1,1 & $39,1 \%$ & $54,9 \%$ & $87,5 \%$ \\
\hline \multirow{5}{*}{$\begin{array}{c}\text { San } \\
\text { Miguel } \\
\text { (Desam- } \\
\text { parados) }\end{array}$} & 5 & $\begin{array}{l}\text { Calera } \\
\text { Monge }\end{array}$ & 66,9 & 0,9 & 4,6 & $17,3 \%$ & $38,9 \%$ & $89,0 \%$ \\
\hline & 6 & $\begin{array}{c}\text { Roy } \\
\text { Villalobos }\end{array}$ & 76,3 & 0,7 & 5,3 & $15,3 \%$ & $30,9 \%$ & $93,4 \%$ \\
\hline & 7 & Romero & 69,4 & 0,6 & 1,8 & $11,5 \%$ & $30,6 \%$ & $94,1 \%$ \\
\hline & 8 & $\begin{array}{l}\text { Quebrada } \\
\text { Honda }\end{array}$ & 77,1 & 0,6 & 0,7 & $22,5 \%$ & $44,3 \%$ & $93,7 \%$ \\
\hline & 9 & Patarrá & 87,4 & 0,5 & 1,0 & $0,0 \%$ & $0,3 \%$ & $98,7 \%$ \\
\hline \multirow{5}{*}{$\begin{array}{c}\text { San } \\
\text { Miguel } \\
\text { (Cartago) }\end{array}$} & 10 & $\begin{array}{c}\text { Los } \\
\text { Ángeles } 1\end{array}$ & 76,1 & 0,5 & 1,2 & $13,3 \%$ & $37,9 \%$ & $90,5 \%$ \\
\hline & 11 & $\begin{array}{c}\text { Los } \\
\text { Ángeles } 2\end{array}$ & 64,3 & 0,4 & 4,6 & $14,1 \%$ & $30,9 \%$ & $96,5 \%$ \\
\hline & 12 & Bermejo & 72,7 & 0,5 & 1,1 & $37,0 \%$ & $56,2 \%$ & $96,0 \%$ \\
\hline & 13 & $\begin{array}{l}\text { Carbocal- } \\
\quad \text { cio }\end{array}$ & 78,1 & 0,6 & 1,6 & $0,0 \%$ & $3,8 \%$ & $96,8 \%$ \\
\hline & 14 & Alvarado & 61,2 & 0,5 & 5,0 & $51,2 \%$ & $75,8 \%$ & $92,2 \%$ \\
\hline Honduras & 15 & Incal & 58,7 & 3,1 & 11,5 & $0,1 \%$ & $0,1 \%$ & $95,6 \%$ \\
\hline Guatemala & 16 & $\begin{array}{c}\text { Cal } \\
\text { importada } \\
2\end{array}$ & 85,1 & 0,3 & 4,4 & $0,0 \%$ & $6,1 \%$ & $88,8 \%$ \\
\hline
\end{tabular}


de $95 \%$ para la suma de hidróxido de calcio e hidróxido de magnesio y la muestra importada de Honduras (cal 15) fue la que presentó el menor porcentaje de hidróxido de calcio. Además, la muestra importada de Honduras y la proveniente de la calera Roy Villalobos presentaron valores de dióxido de carbono mayores que el $5 \%$ permitido por la norma.

Manteniendo pocas diferencias con los resultados obtenidos para la composición química, las muestras de cal que presentaron las granulometrías más adecuadas (según se indica en la norma ASTM C110-11) fueron: cal 1, 3, 9, 13, 15 y 16. Todas las cales mencionadas mostraron valores de $0 \%$ retenido en la malla 30 , por lo que cumplen con las especificaciones de la norma, y hasta $8 \%$ retenido para la malla 200 .

Se realizó además la prueba de retención de agua de la cal. Según la norma ASTM C110-11 (sección 7.1.1), se indica que medir la retención de agua de la cal hidratada es un indicativo de la trabajabilidad que se podrá esperar para el mortero que contenga dicha cal. En la mayoría de los casos seleccionados para el estudio, se tiene cal con retención de agua cercana al $100 \%$ por lo que, al agregar cal hidratada a la mezcla del mortero, se está contribuyendo a que la mezcla de mortero tenga mejor retención y, por ende, mejor trabajabilidad por mayor tiempo.
En el segundo muestreo se obtuvo un caso que apenas cumple con el porcentaje mínimo de $95 \%$ de la suma de hidróxido de calcio y de magnesio indicado en la norma ASTM C20706 (95,1\% en Industrias de Calcio), el resto de cales no cumplen con este requisito. En cuanto al dióxido de carbono, se obtuvieron variaciones que hacen que se sobrepase el 5\% máximo establecido en la norma.

Con respecto a la granulometría, se puede observar que los valores obtenidos siguen cumpliendo con la norma.

La Tabla 5 presenta los porcentajes de variación de obtenidos para las pruebas químicas y granulometría, entre los dos muestreos realizados. Se puede observar que se presentan diferencias importantes entre ambos muestreos en cuanto a la granulometría y los contenidos de hidróxido de magnesio y dióxido de carbono. Por el contrario, se encontró que los contenidos de hidróxido de calcio no presentaron variaciones grandes.

\subsection{Pruebas al mortero con y sin cal}

Se utilizaron los productos de las caleras seleccionadas en el primer muestreo que obtuvieron los contenidos de hidróxido de calcio que se acercaban más a los requisitos de la norma

Tabla 4. Resultados de pruebas a la cal para el segundo muestreo de las caleras visitadas.

\begin{tabular}{|c|c|c|c|c|c|c|c|}
\hline \multirow[b]{2}{*}{ Formación } & \multirow[b]{2}{*}{$\#$} & \multirow{2}{*}{ Calera } & \multicolumn{3}{|c|}{ Pruebas químicas } & \multicolumn{2}{|c|}{ Porcentajes retenidos } \\
\hline & & & $\mathrm{Ca}(\mathrm{OH})_{2}$ & $\mathrm{Mg}(\mathrm{OH})_{2}$ & $\mathrm{CO}_{2}$ & Ret. 30 & Ret. 200 \\
\hline \multirow{3}{*}{ Barra Honda } & 1 & Industrias de & 94,8 & 0,3 & 2,2 & $0 \%$ & $1 \%$ \\
\hline & & Calcio & & & & & \\
\hline & 3 & Nicoyana & 93,2 & 1,0 & 1,9 & $0 \%$ & $2 \%$ \\
\hline $\begin{array}{l}\text { San Miguel } \\
\text { (Desamparados) }\end{array}$ & 9 & Patarrá & 85,7 & 0,7 & 5,5 & $0 \%$ & $0 \%$ \\
\hline San Miguel (Cartago) & 13 & Carbocalcio & 78,5 & 1,0 & 7,3 & $0 \%$ & $5 \%$ \\
\hline
\end{tabular}


Tabla 5. Porcentajes de variación entre el primer y segundo muestreo.

\begin{tabular}{cccccc}
\hline \multirow{2}{*}{ Calera } & \multicolumn{3}{c}{ Pruebas químicas } & \multicolumn{2}{c}{ Porcentajes retenidos } \\
\cline { 2 - 6 } & $\mathrm{Ca}(\mathrm{OH}) 2$ & $\mathrm{Mg}(\mathrm{OH}) 2$ & $\mathrm{CO} 2$ & Ret. 30 & Ret. 200 \\
\hline Industrias de Calcio & $8 \%$ & $-174 \%$ & $-124 \%$ & $29 \%$ & $20 \%$ \\
Nicoyana & $2 \%$ & $90 \%$ & $-12 \%$ & $176 \%$ & $385 \%$ \\
Patarrá & $-2 \%$ & $23 \%$ & $83 \%$ & $28 \%$ & $2248 \%$ \\
Carbocalcio & $1 \%$ & $43 \%$ & $78 \%$ & $7 \%$ & $20 \%$ \\
\hline
\end{tabular}

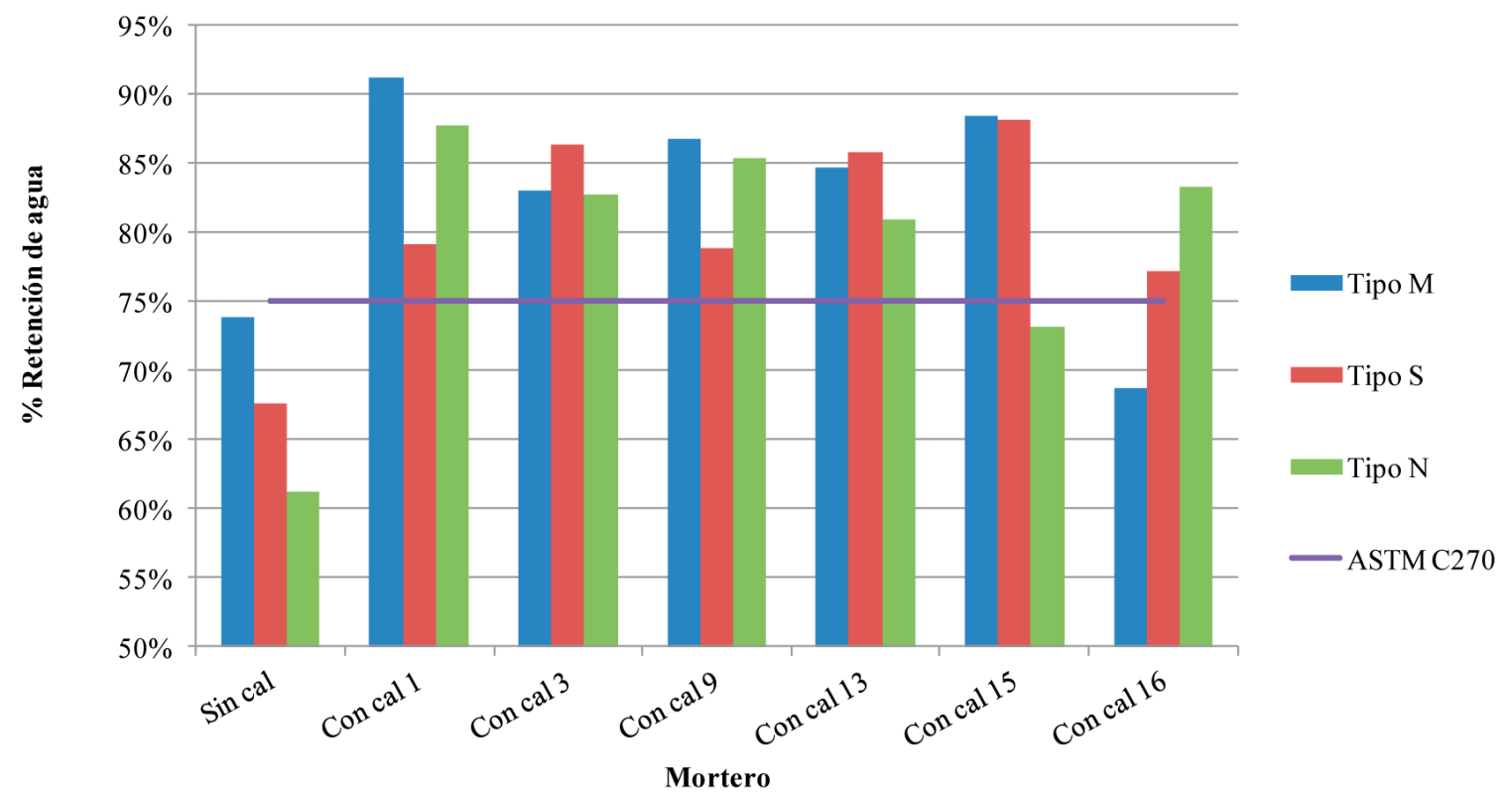

Figura 1. Comparación de resultados de retención de agua del mortero con y sin cal. 


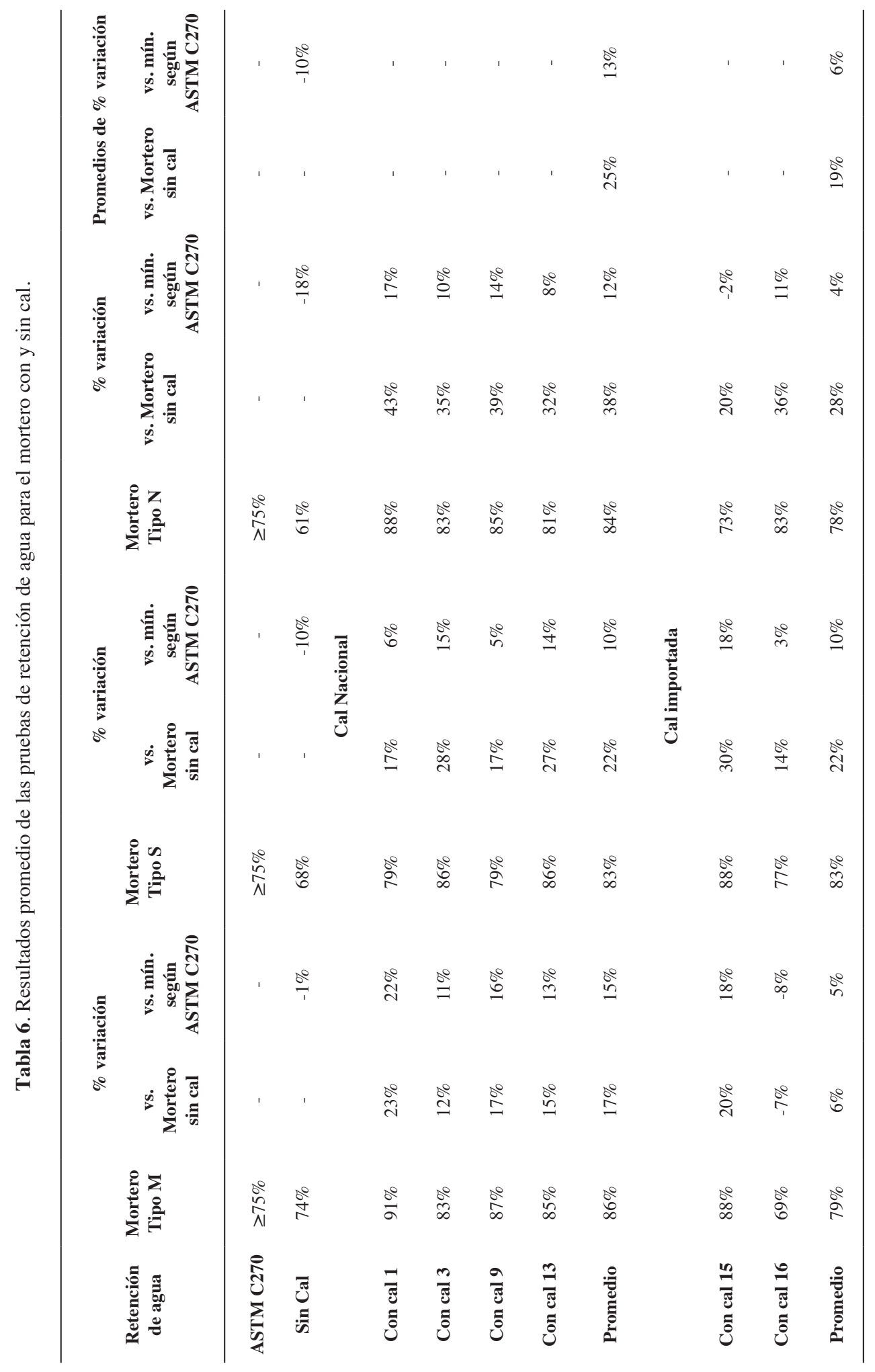


ASTM C207 para su uso en las mezclas de los morteros (marcadas en gris en la Tabla 3). A continuación se muestran los resultados en la Tabla 6 para la prueba de retención de agua, en la Tabla 7 para contenido de aire y en las Tablas 8 a la 10 para la prueba de resistencia a compresión a $\operatorname{los} 7,28$ y 56 días respectivamente.

Se observa en la Tabla 6 que las pruebas de retención de agua realizadas al mortero sin cal presentaron los resultados de un valor menor al $75 \%$ requerido por la norma en todos los casos (mortero tipo M, S y N). Por otro lado, para los morteros con cal nacional se puede verificar que todos los promedios fueron valores mayores al $75 \%$ mínimo. Estos resultados obtenidos para la mezcla de mortero con cal nacional concuerdan con un estudio realizado por Bosiljkov (2001) que también obtuvo un rango del porcentaje de retención de agua del mortero entre $80 \%$ y $90 \%$.

En cuanto a los porcentajes de variación para el mortero con cal nacional con respecto al mortero sin cal, se presentan en la Tabla 6 valores que van desde un $12 \%$ hasta un $43 \%$ de mejora; en promedio, la cal nacional en la mezcla para el mortero mejoró hasta un $25 \%$ la retención de agua con respecto al mortero sin cal.Por otro lado, si bien la cal importada presentó mejoras en muchos casos, también hubo valores de retención de agua menores que los obtenidos para el mortero sin cal por lo que el promedio de mejora en la retención de agua fue solo de $19 \%$.

Comparando los resultados en la Tabla 6 con el porcentaje mínimo de retención de agua proporcionado por la norma ASTM C270 de un $75 \%$, se reafirma que los morteros con cal nacional presentan mejoras en esta propiedad. Los morteros con cal importada también presentan mejoras en la retención de agua, no obstante, el beneficio es un poco menor. Además, se observa como el mortero sin cal aun no alcanza el mínimo de la norma por una diferencia de $10 \%$.

En la Figura 1, se presenta gráficamente la tendencia de mejoras en la retención de agua del mortero al utilizar la cal. El mortero sin cal es el que presenta en la mayoría de los casos los valores más bajos para esta propiedad y, en contraste, en ningún caso de la Figura 1 se tiene que el mortero con cal nacional presente valores menores que los establecidos en la norma ASTM C270.

Para el contenido de aire, el parámetro que establece la norma ASTM C270 es de un máximo de $7 \%$ para morteros tipo M, S y N, para lo cual los resultados obtenidos se encuentran por debajo. Se tiene que el mortero con cal importada fue el que presentó el mayor porcentaje en contenido de aire y el mortero sin cal fue el que presentó menor porcentaje (ver Figura 2). En la Tabla 7 se

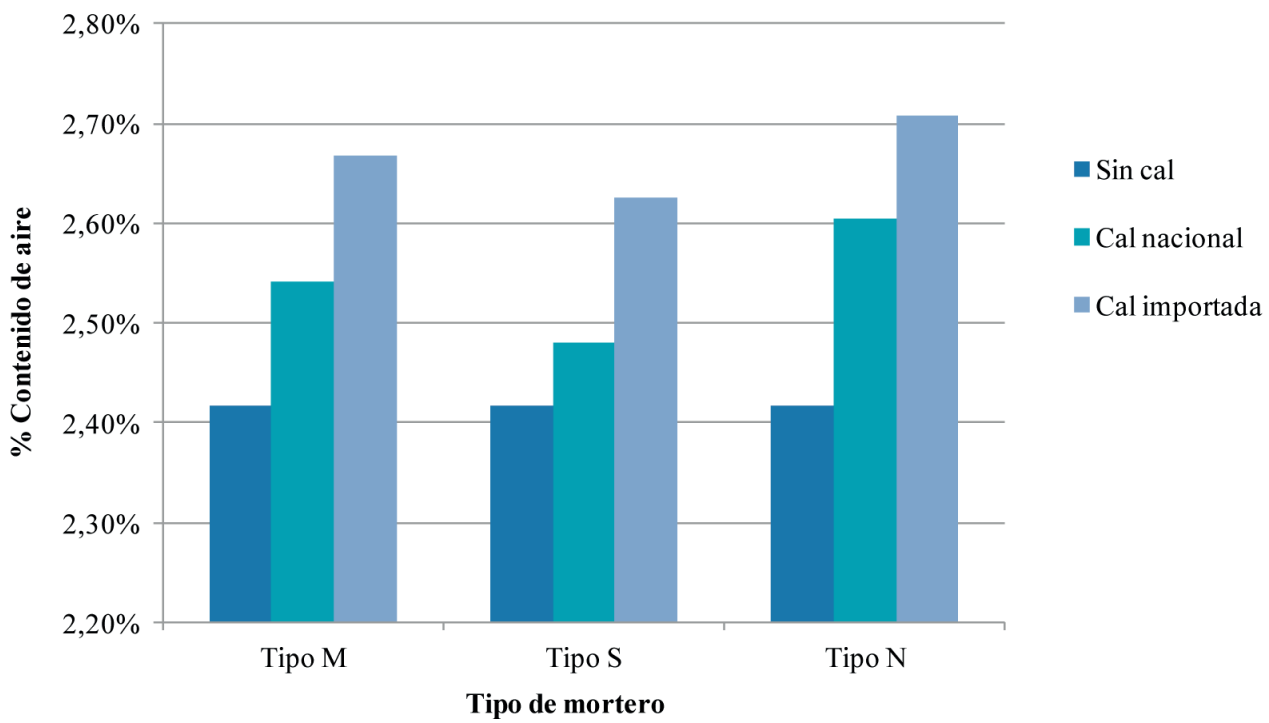

Figura 2. Comparación de resultados de contenido de aire del mortero con y sin cal. 


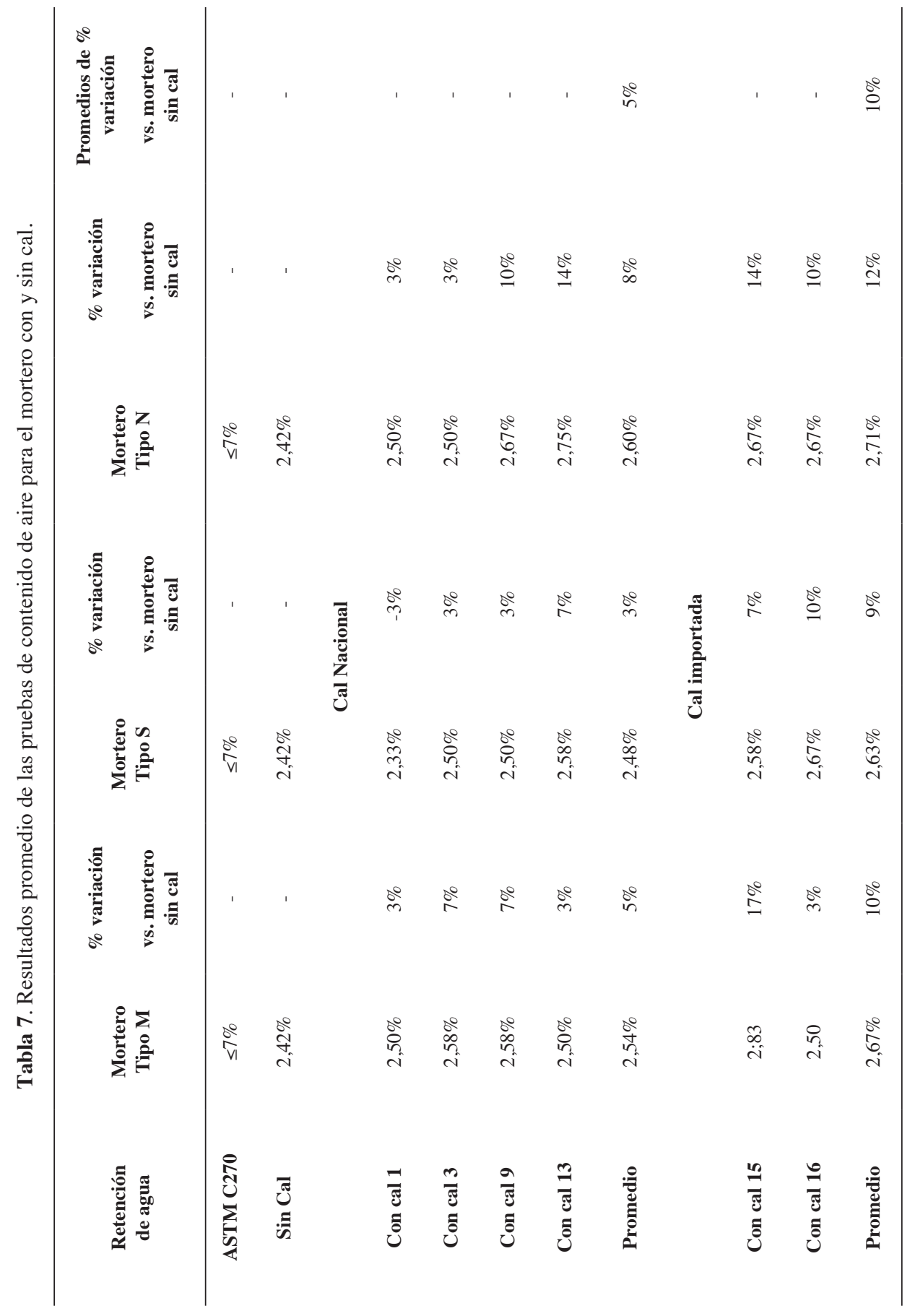




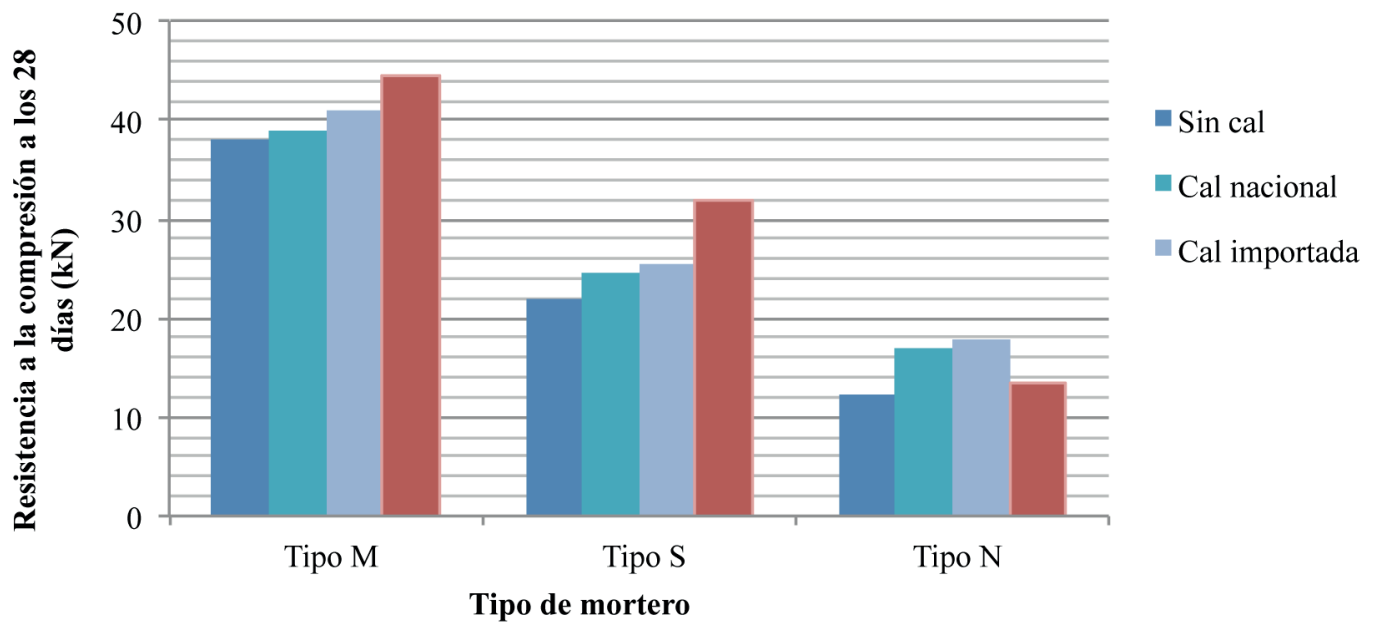

Figura 3. Comparación de los promedios de los resultados de resistencia a la compresión a los 28 días del mortero con y sin cal.

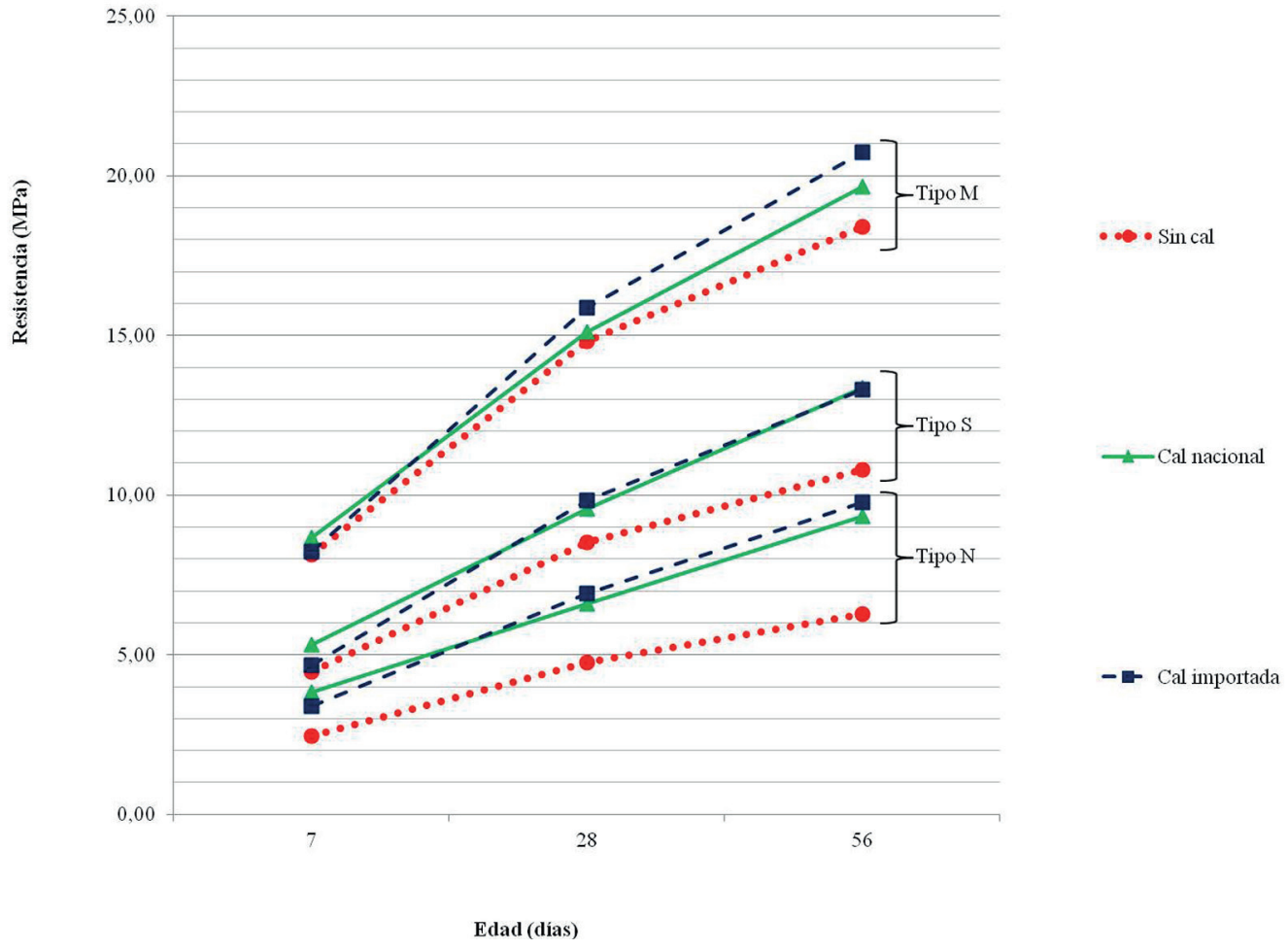

Figura 4. Comparación de resultados de resistencia a la compresión del mortero con y sin cal. 
Tabla 8. Resultados de las pruebas al mortero con y sin cal para resistencia a compresión a los 7 días.

\begin{tabular}{|c|c|c|c|c|c|c|c|}
\hline Mortero & $\begin{array}{c}\text { Tipo M } \\
\text { (MPa) }\end{array}$ & $\begin{array}{l}\text { \% variación } \\
\text { vs. Mortero } \\
\text { sin cal }\end{array}$ & $\begin{array}{l}\text { Tipo S } \\
\text { (MPa) }\end{array}$ & $\begin{array}{l}\text { \% variación } \\
\text { vs. Mortero } \\
\text { sin cal }\end{array}$ & $\begin{array}{l}\text { Tipo N } \\
\text { (MPa) }\end{array}$ & $\begin{array}{l}\text { \% variación } \\
\text { vs. Mortero } \\
\text { sin cal }\end{array}$ & $\begin{array}{c}\text { Promedios de } \\
\text { variación } \\
\text { vs. Mortero sin } \\
\text { cal }\end{array}$ \\
\hline Sin cal & 8,1 & - & 4,5 & - & 2,4 & - & - \\
\hline \multicolumn{8}{|c|}{ Cal Nacional } \\
\hline Con cal 1 & 8,0 & $-1 \%$ & 5,1 & $15 \%$ & 4,1 & $69 \%$ & - \\
\hline Con cal 3 & 9,4 & $16 \%$ & 6,1 & $37 \%$ & 3,6 & $47 \%$ & - \\
\hline Con cal 9 & 8,7 & $7 \%$ & 5,1 & $14 \%$ & 3,3 & $34 \%$ & - \\
\hline Con cal 13 & 8,5 & $4 \%$ & 4,9 & $10 \%$ & 4,3 & $75 \%$ & - \\
\hline Promedio & 8,7 & $6 \%$ & 5,3 & $19 \%$ & 3,8 & $56 \%$ & $27 \%$ \\
\hline \multicolumn{8}{|c|}{ Cal importada } \\
\hline Con cal 15 & 9,3 & $14 \%$ & 5,1 & $14 \%$ & 3,9 & $60 \%$ & - \\
\hline Con cal 16 & 7,2 & $-12 \%$ & 4,3 & $-4 \%$ & 2,8 & $17 \%$ & - \\
\hline Promedio & 8,2 & $1 \%$ & 4,7 & $5 \%$ & 3,4 & $38 \%$ & $15 \%$ \\
\hline
\end{tabular}

observa claramente que el mortero con cal nacional presentó un contenido de aire mayor en un 5\% con respecto al mortero sin cal y que el mortero con cal importada presentó un contenido de aire mayor en un $10 \%$ con respecto al mortero sin cal. Debido a que sí se obtiene un pequeño porcentaje mayor de contenido de aire en morteros con cal con respecto a morteros sin cal, es posible obtener una mejor trabajabilidad de la mezcla cuando se adiciona cal.

Se puede observar en la Figura 4 que en todos los casos y edades los morteros sin adición de cal, ya sea nacional o importada, son los que presentan menor resistencia. La norma ASTM C270 presenta el parámetro de resistencia a la compresión a los 28 días que recomienda como mínimo para la resistencia del mortero. En la Figura 4 se observa que solo los morteros tipo $\mathrm{N}$ con cal nacional y cal importada son los que sobrepasan el mínimo indicado por la norma.
Debido a que la arena utilizada es de tipo industrial, se estuvo trabajando con partículas más quebradas y con más superficies (arena muy angular), lo cual disminuye la trabajabilidad del mortero por utilizar. Por esta razón, se tuvo la necesidad de utilizar mayor cantidad de agua en la mezcla del mortero para mejorar la trabajabilidad, lo cual aumentó la relación agua-cemento y redujo los resultados de resistencia.

Al observar la Tabla 8 a la 10, es posible identificar diferencias en el aporte a la resistencia, con respecto a la cal nacional y la cal importada; sin embargo, se obtiene que no hay ninguna tendencia. Por ejemplo, a los 7 días la cal nacional presenta mejores resultados pero a los 28 días la que presenta mejores resultados es la cal importada, y luego, para la resistencia obtenida a los 56 días, se tienen valores muy similares entre sí para el mortero con cal nacional y el mortero 
NAVAS Y ACÓN: Influencia de la cal en el mortero...

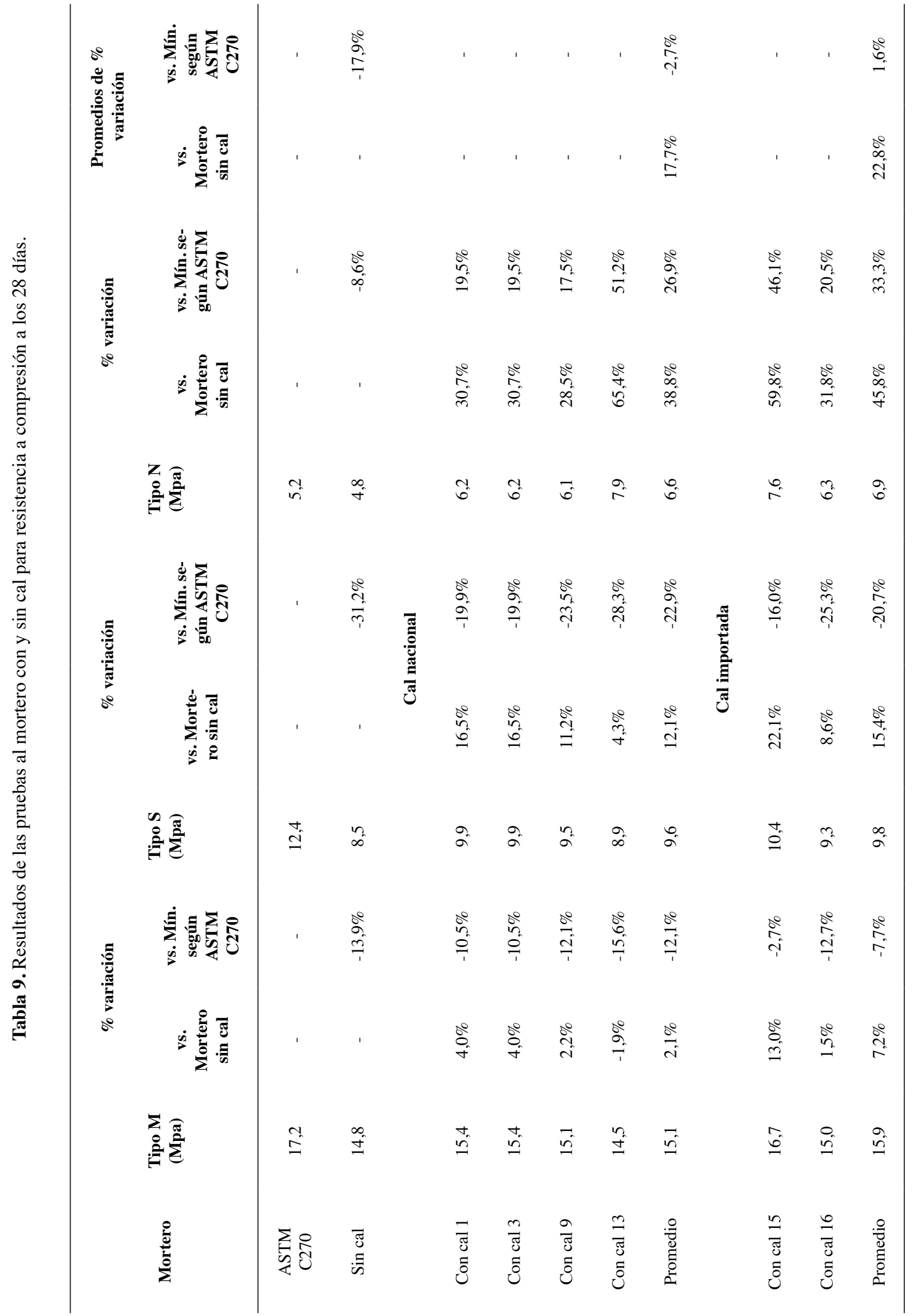




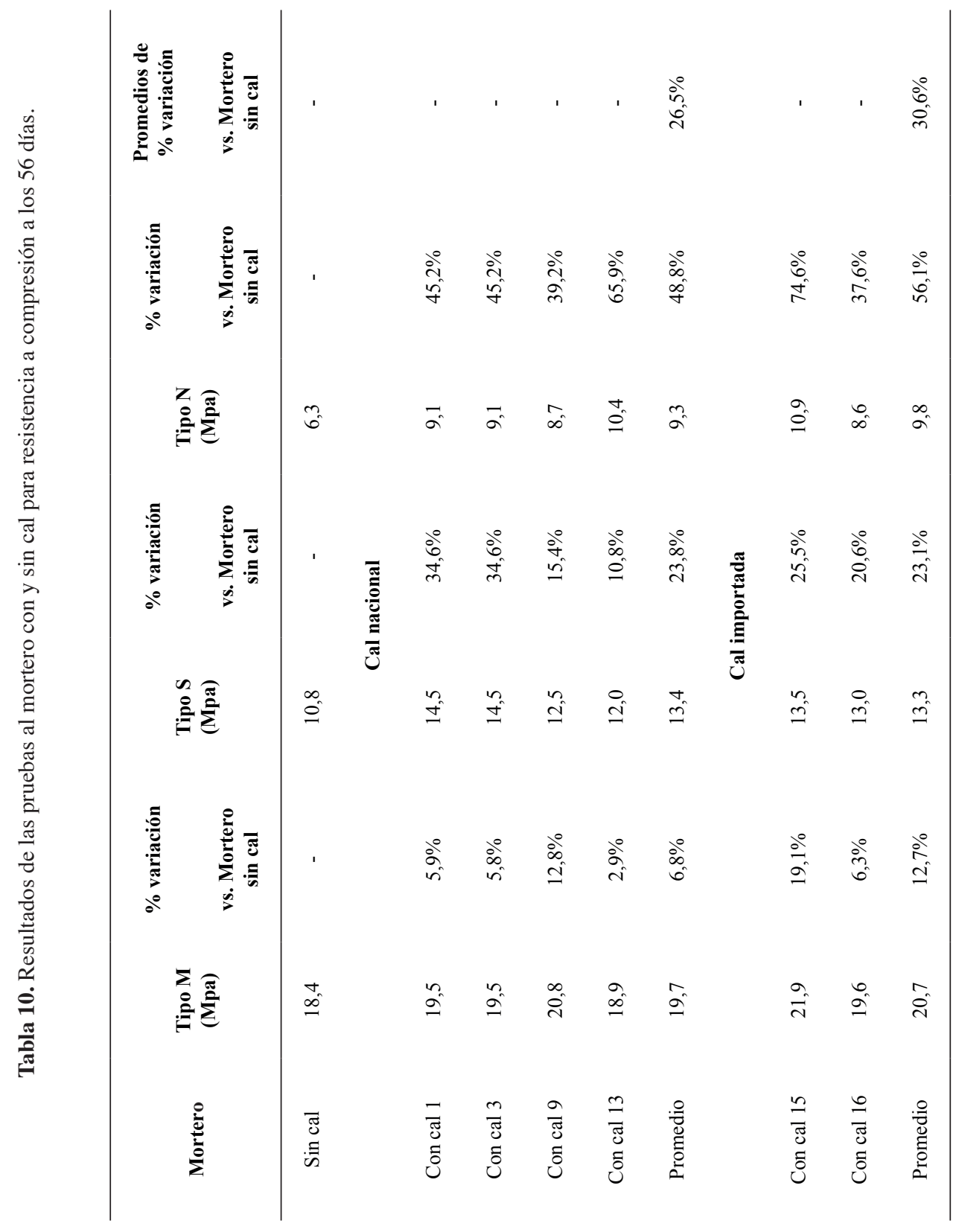


Tabla 11. Porcentajes promedio de variabilidad de la resistencia a diferentes edades de morteros con cal nacional e importada con respecto a morteros fabricados sin cal.

\begin{tabular}{lcc}
\hline & Cal nacional & Cal importada \\
\hline 7 días & $27 \%$ & $15 \%$ \\
28 días & $18 \%$ & $23 \%$ \\
56 días & $27 \%$ & $31 \%$ \\
Promedio & $24 \%$ & $23 \%$ \\
\hline
\end{tabular}

Tabla 12. Porcentajes promedio de variabilidad de la resistencia a diferentes edades de morteros con cal importada con respecto a morteros fabricados con cal nacional.

\begin{tabular}{lc}
\hline & Cal importada \\
\hline 7 días & $-9 \%$ \\
28 días & $4 \%$ \\
56 días & $3 \%$ \\
\hline Promedio & $-0,5 \%$ \\
\hline
\end{tabular}

con cal importada (ver Tabla 12). En todo caso, de las Figuras 3 y 4 y la Tabla 11 sí es posible indicar que la cal aportó mejoras en los valores de resistencia a la compresión con respecto al mortero sin cal.

A los 56 días se mantiene la tendencia en que los morteros con cal, ya sea nacional o importada, presentan resistencias mayores a los morteros sin cal. Por ejemplo, en el caso del mortero con la cal 13 para el tipo $\mathrm{N}$ se tiene una mejora de hasta un $66 \%$, y para el mortero con cal 15 se obtiene un aumento de un $75 \%$.

La Figura 3 resume los resultados de resistencia a la compresión a los 28 días de los diferentes tipos de mortero y la resistencia requerida según la norma ASTM C270. Se observa que los morteros tipo M y S no alcanzaron el valor mínimo establecido por la norma. En el caso de los morteros tipo $\mathrm{N}$, los morteros con cal nacional e importada si cumplieron con este requisito, no así los morteros sin cal.

La Figura 4 muestra las curvas de desarrollo de resistencia para los diferentes morteros. Como se mencionó anteriormente, es posible observar que para todos los tipos de morteros, los morteros sin cal presentan las resistencias más bajas. Las Tablas 11 y 12 muestran un resumen de la variabilidad en la resistencia de los morteros a diferentes edades.

La Tabla 11 contiene las diferencias promedio obtenidas de los morteros fabricados con cal nacional e importada en comparación con los morteros fabricados sin cal. En promedio, se obtuvieron incrementos de $24 \%$ y $23 \%$ en la resistencia de los morteros fabricados con cal nacional e importada respectivamente. 
La Tabla 12 muestra las diferencias de resistencias obtenidas de los morteros fabricados con cal importada con respecto a los morteros fabricados con cal nacional. Se aprecia que no hay una clara tendencia, pues inicialmente (7 días) la cal nacional generó mejores resultados, sin embargo, a los 28 y 56 días se obtuvieron mejoras en los morteros con cal importada. El promedio general de variabilidad fue muy bajo, lo que indica que las resistencias obtenidas con los morteros fabricados con cal nacional e importada son muy similares.

\section{CONCLUSIONES}

Con base en el análisis de los resultados obtenidos y la investigación realizada, se concluye lo siguiente:

\section{Retención de agua}

- La capacidad de retención de agua del mortero con cal nacional se vio mejorada, pues aumentó hasta en un $25 \%$ en promedio, con respecto al mortero sin cal y llegó a cumplir con el mínimo de $75 \%$ indicado por la norma ASTM C270.

- La retención de agua del mortero sin cal no logró sobrepasar el mínimo del $75 \%$ requerido por la norma ASTM C270. Se obtuvieron valores hasta $18 \%$ menores que los establecidos en la norma.

- El uso de cal importada en la mezcla de mortero para pega mejoró la retención de agua en menos de un $20 \%$, en comparación con la retención que presentó el mortero sin cal.

\section{Contenido de aire}

- El contenido de aire de los morteros a los que se les añadió cal hidratada (nacional o importada) aumentó con respecto a los morteros sin cal. Se obtuvo una diferencia de un 5\% entre usar cal nacional o cal importada, con respecto a morteros fabricados sin cal.

- La cal nacional mejoró el contenido de aire del mortero, pues se obtuvo un incremento de un $5 \%$ con respecto a lo obtenido para el mortero sin cal y a su vez los porcentajes se mantuvieron por debajo del máximo permitido por la norma.

- La cal importada aumentó en un $10 \%$ el contenido de aire con respecto a los morteros sin cal y al mismo tiempo no sobrepasó el máximo establecido en la norma.

\section{Resistencia a la compresión}

- Para la resistencia a compresión a los 7,28 y 56 días, siempre se obtuvo menor resistencia en los morteros sin cal.

- Los morteros con cal nacional mostraron un incremento promedio de un $24 \%$, mientras que los morteros con cal importada generaron un incremento promedio de un $23 \%$, con respecto a los morteros sin cal.

- Se obtuvo una diferencia promedio de un $0,5 \%$ entre la resistencia de los morteros con cal nacional y los morteros con cal importada.

- La resistencia a los 28 días de los morteros tipo $\mathrm{M}$ y tipo $\mathrm{S}$ no cumple con el mínimo establecido por la norma ASTM C270.

- Los morteros tipo $\mathrm{N}$ fabricados con cal nacional e importada si cumplieron con la mínima resistencia requerida por la norma a los 28 días.

- Los morteros elaborados sin cal no cumplieron con la resistencia a la compresión a los 28 días establecida por la ASTM C270 en ninguno de los casos.

\section{Generales}

- Se presentaron variaciones en la composición química entre las cales obtenidas en diferentes épocas (verano e invierno).

- A pesar de las variaciones en la composición química de la piedra caliza de las diferentes zonas del país, se pudo obtener beneficios al utilizar cualquiera de las cales en la mezcla para el mortero de pega.

- Fue posible observar que la cal nacional seleccionada para las pruebas, a pesar de no ser obtenida bajo estándares normados, presentó mejoras en las propiedades de los morteros y en muchos casos incluso presentó mejores resultados que la cal importada: 
se obtuvieron mayores resistencias en los morteros fabricados con cal, mayores porcentajes de retención de agua y mayor contenido de aire, por lo que los morteros con cal presentan una mayor resistencia a la compresión, plasticidad y trabajabilidad.

- Conforme se aumenta la relación cal hidratada vs. agregado fino (pasando del mortero tipo $\mathrm{M}$ hasta el mortero tipo N), se van obteniendo aumentos mayores de las diferencias en las propiedades (retención de agua, contenido de aire y resistencia a la compresión) entre los morteros con cal y los morteros sin cal.

\section{RECOMENDACIONES}

A continuación se presentan algunas recomendaciones relacionadas con el trabajo realizado e investigaciones futuras:

- Realizar un mayor número de muestreos a las cales en la época seca y lluviosa, con el fin de estudiar la variabilidad observada en las propiedades químicas en ambas épocas.

- Llevar a cabo pruebas en elementos de mampostería para evaluar y comparar el comportamiento de morteros fabricados con cal.

\section{REFERENCIAS BIBLIOGRÁFICAS}

ACI (American Concrete Institute). (2005). Building Code Requirements for Masonry Structures. ACI 530-05. Michigan: ACI.

ASTM (American Society for Testing and Materials). (2011). ASTM C207-06: Standard Specification for Hydrated Lime for Masonry Purposes. Philadelphia: ASTM.

ASTM (American Society for Testing and Materials). (2011). ASTM C144-11: Standard Specification for Aggregate for Masonry Mortar. Philadelphia: ASTM.

ASTM(American Society for Testing and Materials). (2011). ASTM C25-11: Standard Test Method for Chemical Analysis of Limestone, Quicklime, and Hydrated Lime. Philadelphia: ASTM.
ASTM (American Society for Testing and Materials). (2012). ASTM C109/C109M-12: Standard Test Method for Compressive Strength of Hydraulic Cement Mortars (Using 2-in. or 50mm Cube Specimens). Philadelphia: ASTM.

ASTM (American Society for Testing and Materials). (2011). ASTM C110-11: Standard Test Methods for Physical Testing of Quicklime, Hydrated Lime, and Limestone. Philadelphia: ASTM.

ASTM (American Society for Testing and Materials). (2009). ASTM C1506-09: Standard Test Method for Water Retention of Hydraulic Cement-Based Mortars and Plasters. Philadelphia: ASTM.

ASTM (American Society for Testing and Materials). (2012). ASTM C270-12a: Standard Specification for Mortar for Unit Masonry. Philadelphia: ASTM.

ASTM (American Society for Testing and Materials). (2008). Standard Test Method for Air Content of Hydraulic Cement Mortar. ASTM C185-08 Philadelphia: ASTM.

Bosiljkov, V. (2001). The use of industrial and traditional limes for lime mortars. En Lourenco, P. \& Roca, P. (Eds.) Historical Constructions: Possibilities of Numerical and Experimental Techniques. (págs. 343352). Portugal: Universidad de Minho.

Chacón, C. (2001). Análisis y evaluación técnica de morteros para repello y recomendaciones para su uso. (Proyecto de Graduación para Licenciatura en Ingeniería Civil). Universidad de Costa Rica, San José, Costa Rica.

Picado, A. (2001). Evaluación técnica de morteros para repello y recomendaciones para su aplicación. (Proyecto de Graduación para Licenciatura en Ingeniería Civil). Universidad de Costa Rica, San José, Costa Rica.

Thomson, M. (2000). Plasticity, water retention, soundness and sand carrying capacity: what a mortar needs. En Bartos, P., Groot, C. \& Hughes, J (Eds.). Historic Mortars: Characteristics and Tests. (págs. 163-171). France: Rilem Publications S.A.R.L. 


\section{SOBRE LOS AUTORES}

Alejandro Navas Carro. M. Sc. Ingeniería Civil Profesor de la Escuela de Ingeniería Civil de la Universidad de Costa Rica. Director del Laboratorio Nacional de Materiales y Modelos Estructuras (LANAMME). Apartado Postal 2060,
UCR, San José, Costa Rica. Teléfono: 2511-4186. Correo electrónico: anavas@lanamme.ucr.ac.cr

Catherine M. Acón Villalobos. Lic. Ingeniería Civil. Ingeniera de Proyectos en América Construcciones (AMCO). Celular: 8893-3686. Correo electrónico: aconcatherine@gmail.com 